\title{
Sensorless control for a synchronous reluctance motor based on current oversampling using standard PWM excitation
}

\author{
M. Giuliato ${ }^{1}$, L. Peretti ${ }^{2}$, F. Tinazzi ${ }^{1}$, M. Zigliotto ${ }^{1}$ \\ ${ }^{1}$ Dept. of Management and Engineering, University of Padova, Italy \\ ${ }^{2}$ Division of Electric Power and Energy Systems, KTH Royal Institute of Technology, Stockholm, Sweden \\ *E-mail: \{fabio.tinazzi, mauro.zigliotto\}@unipd.it, lucap@kth.se
}

Keywords: Sensorless control, current oversampling, synchronous reluctance motor

\begin{abstract}
This paper discusses a sensorless control technique for synchronous reluctance machines based on an alternative way to process the information obtained from the stator current oversampling. The mathematical background is presented and demonstrated through experimental validation. Implementation issues are highlighted throughout the paper.
\end{abstract}

\section{Introduction}

Sensorless control is a modern trend in AC motor drives, because it enables cost reduction while improving the overall reliability of the system. A key feature in sensorless motor drives is the presence of significant computational power and of fast analog-to-digital conversion electronics in control hardware boards. This ever-increasing availability paves the way for the implementation of a variety of different position observers or estimators, which can be quite complex and sophisticated as the hardware devices become more powerful.

Among the several alternatives, the trend of extracting information from the current ripple has its charm. Up to recent times, the current ripple was considered mostly an annoying but unavoidable side effect of voltage pulse-width modulation. However, the current ripple does contain useful information for sensorless control purposes. Among the first successful attempts that exploit such information for sensorless control, Nussbaumer et al. [1], [2] date back about 10 years ago.

Since then, other researchers looked into exploiting the current ripple information, particularly using stator current oversampling. One of the first issues that was addressed was the reduction of the magnitude of the injected high-frequency signal used to detect the rotor anisotropic structure (and therefore the rotor position in a synchronous machine). An example of a solution for this issue is discussed in Landsmann et al. [3].

In 2011, Bolognani et al. [4] proposed a sensorless control for an internal permanent magnet synchronous motor (IPM) which exploited Rogowski-type current derivative sensors. The rotor position was retrieved from current oversampling and real-time processing of the measurements.

In 2012, Sumner et al. [5] proposed to approximate the current ripple measured from standard current sensors as a linear piece-wise function. In this application for a permanent magnet synchronous motor (PMSM), the linear profile of the current ripple between two vertices of the piece-wise function was estimated by using a least-square (LS) approximation.

Due to the complexity of obtaining clean and meaningful estimation of the current derivatives, through conventional sensor technology, in 2017 Hind et al. [6] replaced the LS approximation by artificial neural networks (ANN). The current ripple slope was measured in a more precise and reliable way, at the cost of both an increased complexity of the algorithm that required a quite high computational effort.

Again in 2017, Weber et al. [7] presented a reduced-order observer for a PMSM based on a LS regression of the oversampled current measurements during the inverter passive and active switching states. This enabled the estimation of the current slopes and their exploitation for the purposes of rotor position estimation.

In 2019, Chen et al. [8] described an interesting application example of current oversampling for the sensorless control of a PMSM in rail transportation. The paper underlines that the sensorless control approach is highly preferred because a position sensor would suffer of both overtemperatures and mechanical stress when integrated in the traction drive train system.

Other recent works address several different issues of this interesting and relatively recent technique. Bui et al. [9] propose a method for determining the synchronous $d, q$ inductances in a real-time fashion, focusing on the elimination of a shaft-locking mechanism and of a test signal. The algorithm is very rapid because it does not require any recursive algorithm technique. Nevertheless, it does not implement a complete sensorless drive, therefore not fully considering the issues of current derivative implementation during normal operation.

The paired works of Lindemann et al. [10] and Himker et al. [11] introduce an enhanced position observer based on current oversampling for an IPM. The algorithm exploits of both active and passive switching states of an inverter. The rotor position is obtained by comparing predicted and measured current slopes. The tracking speed is enhanced by using an iterative tracker 
instead of a conventional proportional-integral (PI) tracker. An interesting compensation of the time discretization error is also discussed in [10].

The aforementioned research articles are a fertile ground for the study of stator current oversampling aimed at the sensorless control of synchronous reluctance motors (SynRMs), which has not been proposed yet. It is also worth noting that all the presented techniques rely on the measurement of the current derivatives during two or three vectors of the space vector pulse-width modulation (SVPWM). Since the derivative computation is troublesome when the voltage vector is applied for a relatively short time, the algorithms generally require either a modification, of the voltage modulation, or a current injection, or the reconstruction of the missing current derivatives.

The present paper contributes to two issues. The first one is the use of only one state vector for the estimation of the current derivative. The rotor position is estimated by a recursive LS regression on the oversampled currents, only during the longest voltage vector among those applied in a SVPWM period. Neither high frequency current injection nor PWM modification are required. Standard current sensors are used, avoiding the additional cost and hardware circuitry complexity required by Rogowski-coils. The second contribution is relative to the application to a SynRM, an emerging machine which targets low-to-middle end AC drives applications. SynRM sensorless control is almost a must-have, but it is also more complicate to achieve because of the inherent non-linearities of these machines.

The paper is organised as follows. Sect. 2 briefly resumes the mathematical model of the SynRM, while Sect. 3 provides the mathematical background of the position estimation adopted in the sensorless control. Sect. 4 describes the implementation on a laboratory test bench, while Sect. 5 presents some preliminary experimental results obtained from the proposed estimation in an open-loop configuration. Some conclusions wrap the paper up.

\section{The synchronous reluctance machine model}

The SynRM rotor position estimation implemented in this work uses as the mathematical model of a SynRM in the stationary reference frame $\alpha \beta$, which is briefly recalled hereafter. The cross-coupling inductances and the effect of magnetic iron saturation are neglected for the sake of simplicity. The model equation written in a matrix form is as follows:

$$
\begin{aligned}
\boldsymbol{u}_{\alpha \beta} & =R \boldsymbol{i}_{\alpha \beta}+\frac{d \boldsymbol{\lambda}_{\alpha \beta}}{d t} \\
& =R \boldsymbol{i}_{\alpha \beta}+\frac{d \boldsymbol{L}_{\alpha \beta}}{d t} \boldsymbol{i}_{\alpha \beta}+\boldsymbol{L}_{\alpha \beta} \frac{d \boldsymbol{i}_{\alpha \beta}}{d t}
\end{aligned}
$$

where $R$ is the stator resistance, $\boldsymbol{u}_{\alpha \beta}, \boldsymbol{i}_{\alpha \beta}$ and $\boldsymbol{\lambda}_{\alpha \beta}$ are respectively the stator voltage, stator current and the stator flux linkage vectors in the $\alpha \beta$ reference frame. The matrix $\boldsymbol{L}_{\alpha \beta}$ is the inductance matrix in the $\alpha \beta$ reference frame which is expressed as:

$$
\boldsymbol{L}_{\alpha \beta}=\left[\begin{array}{cc}
L_{\Sigma}-L_{\Delta} \cos \left(2 \vartheta_{\mathrm{me}}\right) & -L_{\Delta} \sin \left(2 \vartheta_{\mathrm{me}}\right) \\
-L_{\Delta} \sin \left(2 \vartheta_{\mathrm{me}}\right) & L_{\Sigma}+L_{\Delta} \cos \left(2 \vartheta_{\mathrm{me}}\right)
\end{array}\right]
$$

In (2), $L_{\Sigma}$ and $L_{\Delta}$ are equal to

$$
L_{\Sigma}=\frac{L_{d}+L_{q}}{2} \quad L_{\Delta}=\frac{L_{q}-L_{d}}{2}
$$

where $L_{d}$ and $L_{q}$ are the direct and quadrature synchronous inductance, respectively. This model assumes the knowledge of the stator resistance $R$ (more comment on this in Sect. 5 ), and also assumes symmetry between the three phases of the machine (i.e. the stator resistance is the same for each phase). On the other hand, the inductances $L_{d}$ and $L_{q}$ are not considered as constants.

\section{The proposed estimation based on current derivatives}

The proposed rotor position estimation is performed during each of the eight possible voltage vectors (six active, two zeros) applied during a SVPWM period. The estimation relies on the oversampled measurement of the phase currents. The key aspect is to perform a recursive LS estimation only for the voltage vector that is applied for the longest time interval, in order to leverage on the larger time window for the estimation of the current derivative. A large time window is beneficial because the phase currents are affected by high frequency oscillations whenever one of the power electronics switches in the converter change their state, causing a steep change in the voltage output. This phenomena cannot be classified as noise, since it is originated by parasitic capacitive elements either in the converter or in the machine (or a combination of both), and even due to capacitive coupling of long cables [12]. These oscillations have a negative effect on the recursive LS regression, which increase its convergence time and may be even return incorrect results, eventually. In addition, the selection of the longest applied voltage vector ensures that a minimum pulse violation for the current derivation is practically avoided at any time.

We assume that the drive under analysis is rotating at an electromechanical speed $\omega_{\text {me }}$ which is much lower than the SVPWM frequency used in the SynRM drive. With this assumption, the angular electromechanical position $\vartheta_{\text {me }}$ can be considered as a constant during a single period $T_{\mathrm{PWM}}$ of the SVPWM. Thus, the values of $\cos \left(2 \vartheta_{\text {me }}\right)$ and $\sin \left(2 \vartheta_{\text {me }}\right)$ appearing in the matrix in (2) are considered as constants over the time interval $T_{\mathrm{PWM}}$.

Moreover, due to the ratio between the electrical time constant and the SVPWM period $T_{\mathrm{PWM}}$, the current ripple can be approximated as a linear piece-wise function, where each vertex of the function is located at a time instant where a new voltage vector is applied to the machine. The consequence is that the the current derivatives are considered as constants during the time interval where a single voltage vector is applied.

Due of the aforementioned assumptions, (1) and (2) become a linear system that can be solved for the unknowns $\cos \left(2 \vartheta_{\text {me }}\right)$ and $\sin \left(\vartheta_{\text {me }}\right)$. By using $\widehat{\vartheta}_{\text {me }}$ instead of $\vartheta_{\text {me }}$ to underline the nature of estimation of the angular position, and by solving the mathematical steps involved in the extraction of the variable 
$\widehat{\vartheta}_{\text {me }}$, the expressions that return the estimated electromechanical position are equal to:

$$
\begin{gathered}
\widehat{\vartheta}_{\mathrm{me}}=\frac{1}{2} \arctan \left(\frac{n u m}{d e n}\right) \\
n u m=-\frac{d i_{\beta}}{d t} u_{\alpha}-\frac{d i_{\alpha}}{d t} u_{\beta}+R\left(\frac{d i_{\beta}}{d t} i_{\alpha}+\frac{d i_{\alpha}}{d t} i_{\beta}\right)+ \\
2 L_{\Sigma}\left(\frac{d i_{\beta}}{d t} \frac{d i_{\alpha}}{d t}\right)+2 \omega_{\mathrm{me}}\left[i_{\alpha} u_{\alpha}-i_{\beta} u_{\beta}+\right. \\
\left.R\left(i_{\beta}^{2}-i_{\alpha}^{2}\right)+L_{\Sigma}\left(\frac{d i_{\beta}}{d t} i_{\beta}-\frac{d i_{\alpha}}{d t} i_{\alpha}\right)\right] \\
\operatorname{den}=\frac{d i_{\beta}}{d t} u_{\beta}-\frac{d i_{\alpha}}{d t} u_{\alpha}+R\left(\frac{d i_{\alpha}}{d t} i_{\alpha}-\frac{d i_{\beta}}{d t} i_{\beta}\right)+ \\
L_{\Sigma}\left(\frac{d i_{\alpha}{ }^{2}}{d t}-\frac{d i_{\beta}{ }^{2}}{d t}\right)+2 \omega_{\mathrm{me}}\left[-i_{\alpha} u_{\beta}-\right. \\
\left.i_{\beta} u_{\alpha}-R\left(2 i_{\alpha} i_{\beta}\right)+L_{\Sigma}\left(\frac{d i_{\beta}}{d t} i_{\alpha}+\frac{d i_{\alpha}}{d t} i_{\beta}\right)\right]
\end{gathered}
$$

The set of equations (4)-(6), together with the estimation of the current derivatives, are implemented according to the description reported in Sect. 4.

\section{Implementation and tests}

\subsection{The test bench}

The proposed estimation was implemented and tested in the the experimental setup shown in Fig. 1. The setup is composed by two electrical machines mechanically connected in a backto-back configuration. Each machine is driven by a frequency converter. The SynRM tested in this paper (see its parameters in Table 1) is on the right side of the picture and it is indicated as MUT (machine under test). This machine is driven by a custom frequency converter, with an interface board connected to a DSPACE MicroLabBox system where the field-oriented control of speed and current and the position estimation algorithm are implemented. The DSPACE system allows implementation both at DSP and FPGA levels, both of them have been used for the implementation of the proposed algorithm. The load machine is a synchronous machine driven by an off-the-shelf frequency converter.

The SVPWM implemented in the DSPACE system operates at a switching frequency of $10 \mathrm{kHz}$, and the dead time for the IGBTs is equal to $t_{d}=4 \mu \mathrm{s}$. The phase currents ar measured with LEM current sensors with a frequency bandwidth of $150 \mathrm{kHz}(-1 \mathrm{~dB})$.

\subsection{The implemented algorithm}

An overview of the implementation of the field-oriented control and the position estimation algorithm is given in Fig. 2.

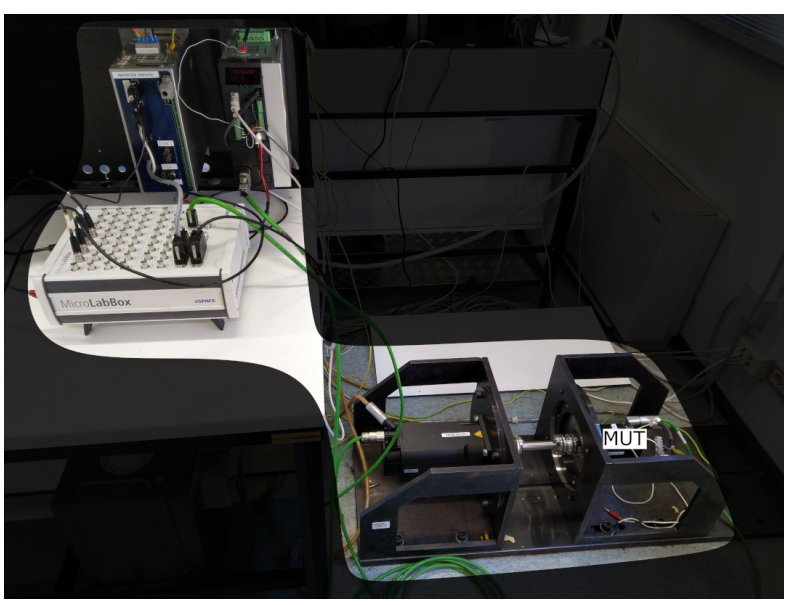

Fig. 1. A picture of the experimental setup.

Table 1 Parameters of the SynRM under analysis.

\begin{tabular}{c|c}
\hline Nominal current & $4 \mathrm{~A}$ \\
Nominal speed & $1500 \mathrm{rpm}$ \\
Nominal torque & $5.5 \mathrm{~N} \mathrm{~m}$ \\
Pole pairs & 2 \\
Stator resistance $\left(\right.$ at $20^{\circ} \mathrm{C}$ ) & $4.76 \Omega$ \\
$d$-axis inductance (linear magnetic region) & $380 \mathrm{mH}$ \\
$q$-axis inductance (linear magnetic region) & $85 \mathrm{mH}$ \\
\hline
\end{tabular}

A more detailed look into the position estimation block of Fig. 2 is shown in Fig. 3. For the sake of a safer initial testing experience, the position estimation was firstly implemented in an open-loop configuration, i.e. using the measured position (obtained by an encoder) to close both current and speed control loops, while comparing the real and estimated angular position. Note that the current derivative block uses a set of currents which are oversampled with respect to the ones used for the position estimation and the field-oriented control. More details are given in the next subsection.

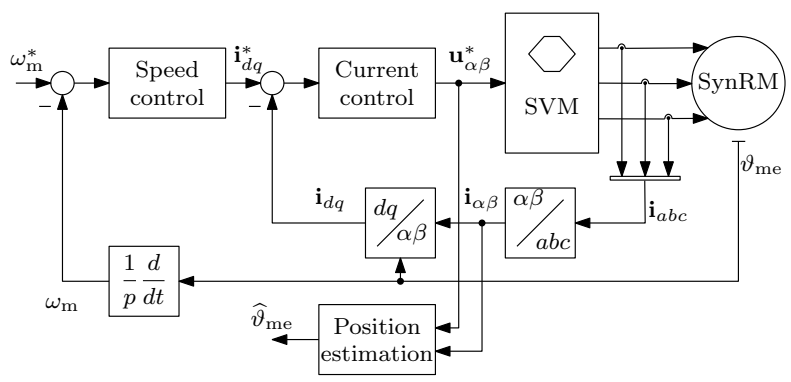

Fig. 2 Schematic for the test of the SynRM drive with position estimation algorithm.

In Fig. 3, the angular position calculation block implements the mathematical steps (4)-(6), and it is preceded by the estimation of the current derivatives which will be described in the next subsection. It is worth remembering that the angular position is estimated with a modulo of $\pi$ electrical radians, because 


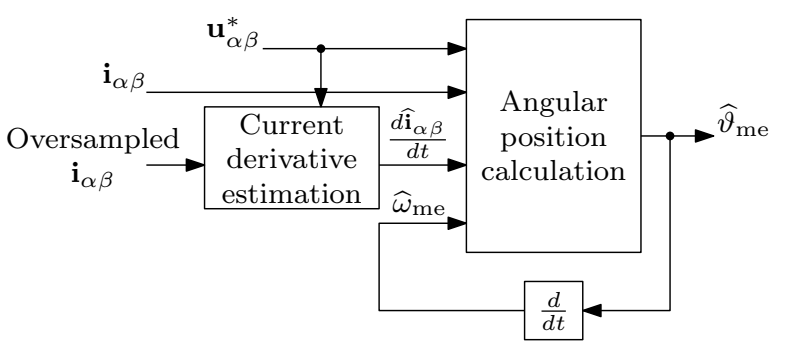

Fig. 3. Schematic of the proposed position estimation.

of the $2 \vartheta_{\text {me }}$ dependency in (4)-(6). However, the use of an initial position algorithm to estimate the initial rotor position removes the $\pi$ uncertainty (see for example [13]).

It is also worth mentioning that the stator resistance $R$ is assumed to be known and updated in real time by an online tracking algorithm, as the one proposed in [14].

\subsection{The current derivative estimation}

The relative importance of the current derivative estimation block requires further details. This operation is performed in the FPGA of the DSPACE system, in advance with respect to the angular position calculation. The schematic of the current derivative estimation block of Fig. 3 is reported in Fig. 4.

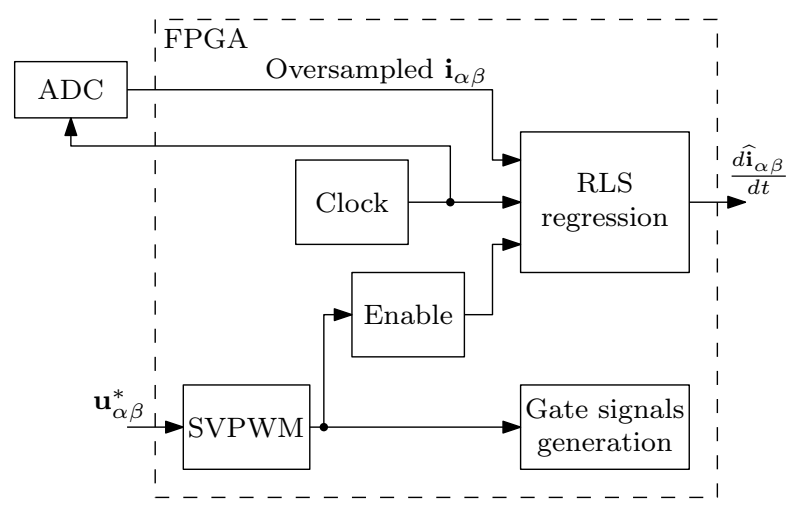

Fig. 4. Schematic of the current derivative estimation.

At the core of the FPGA, a clock is driving an analog-todigital converter (ADC) to perform phase current measurements with an oversampling frequency of $10 \mathrm{MHz}$. For a switching frequency of $10 \mathrm{kHz}$, this ensures the presence of 1000 current samples per switching period. A recursive LS regression of the current ripple slope is implemented based on the oversampled currents, driven by an enable logic signal generated from the SVPWM gate signal output.

The behaviour of the enable logic signal is reported in Fig 5. This logic signal is high during the time interval in which the longest voltage vector is applied. The enable signal excludes the inverter dead time $t_{d}$, and even waits for a little bit more by introducing an estimation dead time called $t_{\text {wait }}$. The time interval $t_{\text {wait }}$ is introduced to let the highfrequency oscillations in the current measurements to decay, thus improving the position estimation accuracy. The next subsection brings more detail on the problem of clean and reliable current measurements.

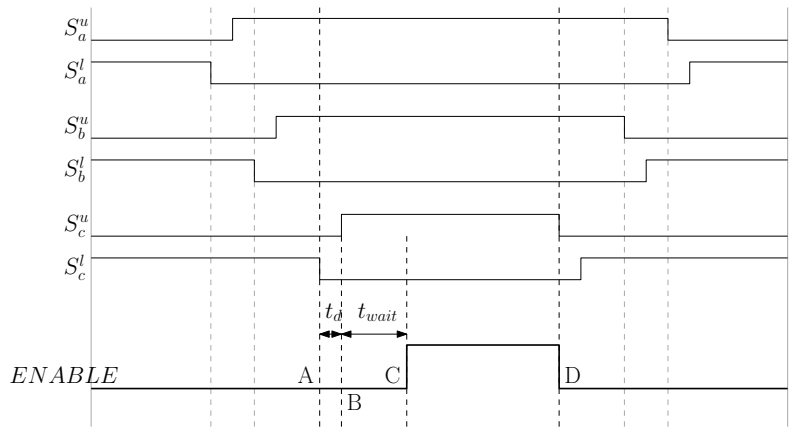

Fig. 5 Generation of the enable signal for the recursive LS regression.

\subsection{Consideration on phase current sampling}

An example of a phase current measurement within a SVPWM period is shown in Fig. 6. The $A B C D$ marks correspond to the instants reported in the enable logic signal timing diagram of Fig. 5.

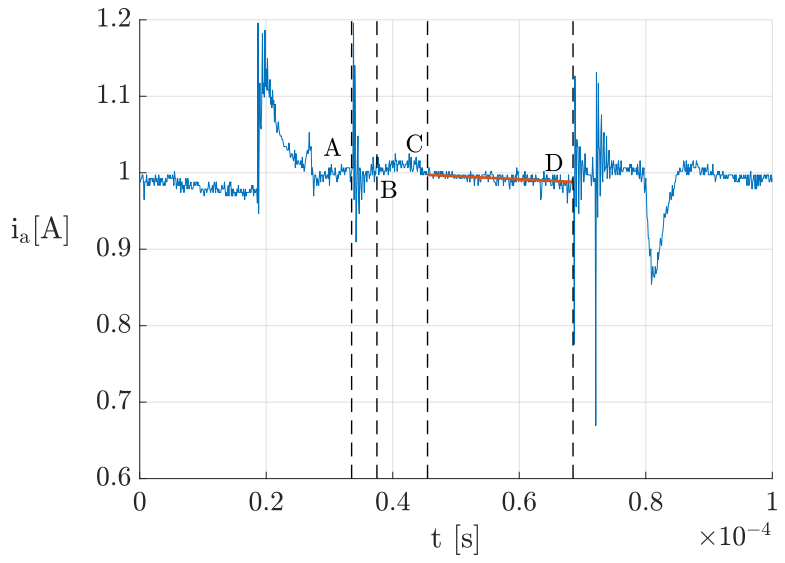

Fig. 6 An example of oversampled phase current with corresponding marks for enable logic signal generation.

Fig. 5 shows that it is not feasible to perform current derivative estimation before the oscillation are extinguished. The segment $C D$ is the longest reliable stretch of measurement, included in the longest time interval of a voltage vector, in which the current slope is estimated.

The presence of high-frequency oscillations, which was earlier mentioned as a consequence of parasitic capacitances, introduces a further issue. If the parasitic capacitances are located in the machine (due to turn-to-ground or inter-turn capacitances), the sum of the three phase currents measured at the inverter output (where the current sensors are typically located) is not necessarily zero when phases are connected in 
star configuration. Thus, if only two currents are measured and the third one is obtained as the opposite of the sum of the first two, it may be possible that oscillations are amplified. This decreases the current derivative estimation as well as the angular position estimation, especially when the derivative estimation is performed during the long application of a zero voltage vector (because of the relatively small current slope).

An example of experimental results obtained measuring either two or three phase currents is reported in Fig. 7. The benefit of measuring three phase currents is visible.
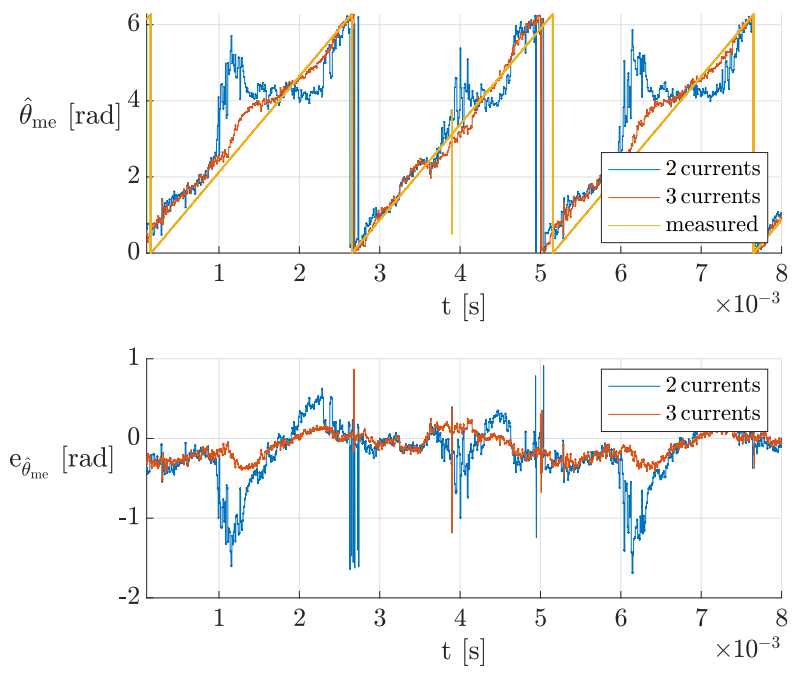

Fig. 7 (top) Estimated and measured rotor position, (bottom) rotor position estimation error at steady state with two or three phase current measurements.

\section{Preliminary experimental results}

Experimental tests at different mechanical speeds at steady state, and during either speed or torque transients, verified the performances of the proposed angular position estimation. Fig. 8 reports the mechanical speed, he estimated and measured electromechanical angle, and the angular position estimation error at a speed of $400 \mathrm{rpm}$ at no load conditions. The angular position error is always less than \pm 0.5 radian.

The experimental results obtained during step variations of the speed reference between 375 and $750 \mathrm{rpm}$, and from 750 back to 375 rpm, are reported in Fig. 9 and Fig. 10, respectively. These experiments validate the estimation accuracy during transients and show that the position estimation error decreases as the speed increases. This is due to the gradual increased dominance at higher speeds of the speed-dependent terms in (5) and (6), which are found to be less affected by noise and high-frequency oscillations.

The torque disturbance rejection capability test of the position estimation is reported in Fig. 11. A step variation of $50 \%$ of the nominal torque is applied at $5 \mathrm{~ms}$, where the current is regulated at half of its nominal value in magnitude. The position estimation is still accurate during the transient conditions.
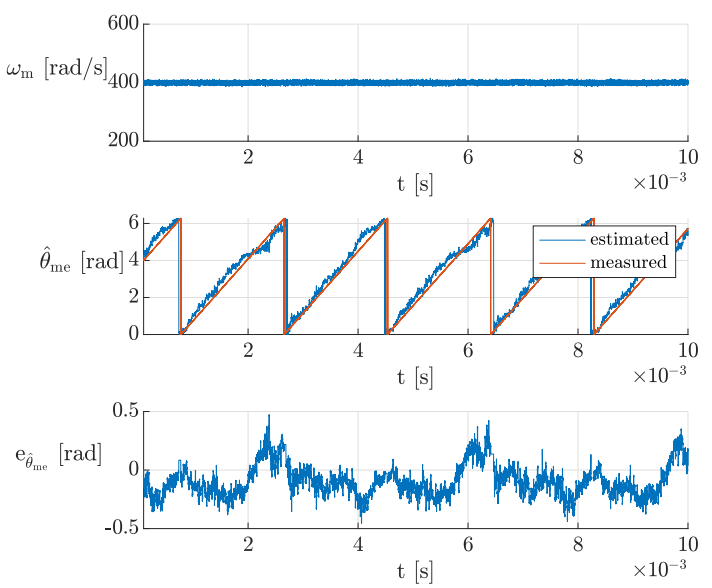

Fig. 8 Measured rotor speed, estimated and measured rotor position, rotor position estimation error at steady state.
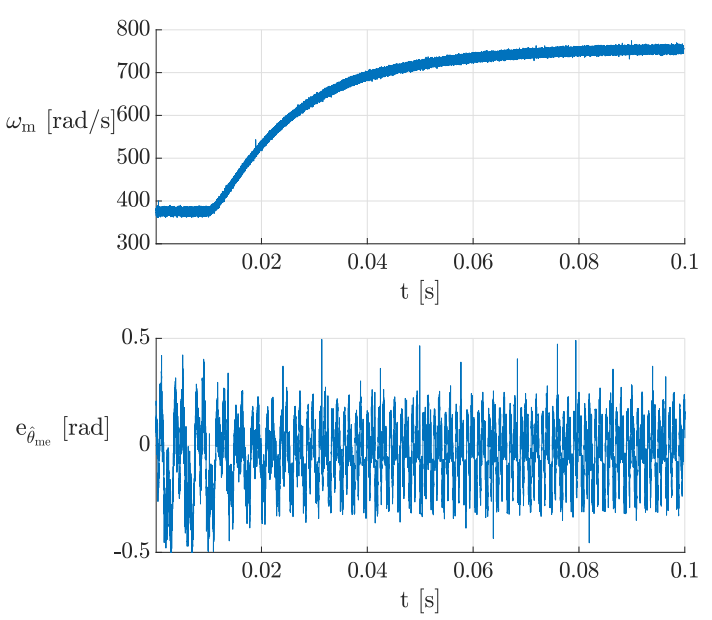

Fig. 9 Measured rotor speed and rotor position estimation error during a step variation of the reference speed from 375 to $700 \mathrm{rpm}$.
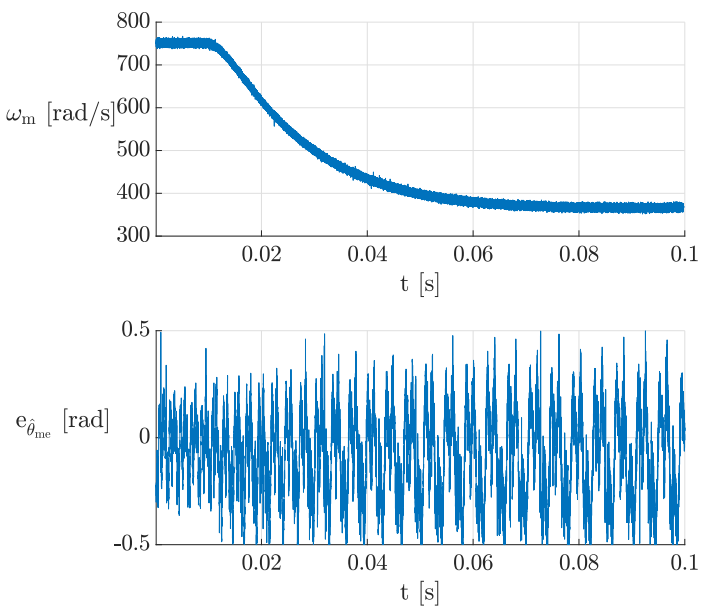

Fig. 10 Measured rotor speed and rotor position estimation error during a step variation of the reference speed from 700 to $375 \mathrm{rpm}$. 

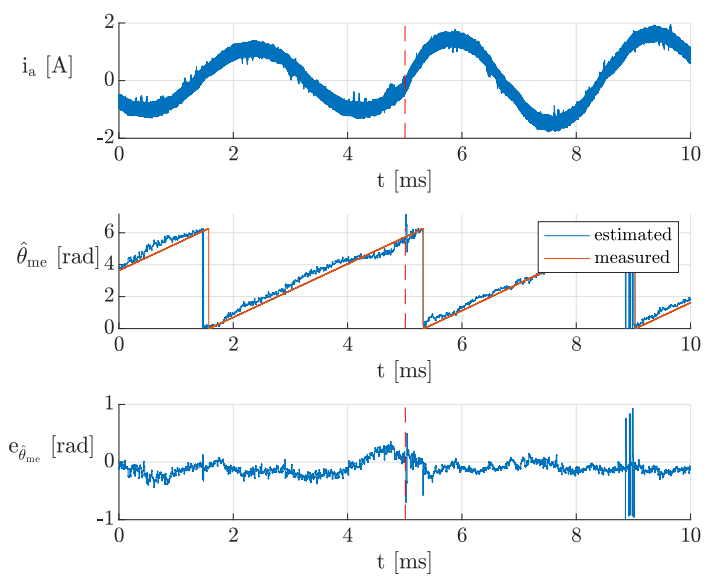

Fig. 11 Phase current, estimated and measured rotor position and rotor position estimation error during a load torque step variation.

\section{Conclusions}

This paper describes an angular position estimation based on oversampled stator currents, and its implementation for sensorless control purposes in a SynRM drive. The experimental validation is performed with the estimation placed in open loop with respect to the field-oriented control. The estimation algorithm does not require any high-frequency current injection or PWM modification, using a recursive least-square algorithm estimation of the current derivatives based on the measurements obtained from a standard current sensor. The derivative estimation is performed on the longest time interval of application of one voltage vector within one SVPWM period. Among the implementation hints, the importance of measuring three phase currents is underlined. Future works will encompass the estimation in closed-loop conditions for the SynRM drive under test.

\section{References}

[1] P. Nussbaumer and T. M. Wolbank, "Using oversampling techniques to extract AC machine saliency information," in Proceedings of the 36th Annual Conference of the IEEE Industrial Electronics Society (IECON), Nov. 2010, pp. 1035-1040.

[2] _ "Using switching transients to exploit sensorless control information for electric machines," in Proceedings of the 2nd IEEE International Symposium on Sensorless Control for Electrical Drives (SLED), Sep. 2011, pp. 35-40.

[3] P. Landsmann, J. Jung, M. Kramkowski, P. Stolze, D. Paulus, and R. Kennel, "Lowering injection amplitude in sensorless control by means of current oversampling," in Proceedings of the 3rd IEEE International Symposium on Sensorless Control for Electrical Drives (SLED), Sep. 2012, pp. 1-6.

[4] S. Bolognani, S. Calligaro, R. Petrella, and M. Sterpellone, "Sensorless control for IPMSM using PWM excitation: analytical developments and implementation issues," in Proceedings of the 2nd Symposium on Sensorless Control for Electrical Drives (SLED), Sep. 2011, pp. 64-73.

[5] Y. Duan and M. Sumner, "A novel current derivative measurement using recursive least square algorithms for sensorless control of permanent magnet synchronous machine," in Proceedings of the 7th International Power Electronics and Motion Control Conference (PEMC), vol. 2, Jun. 2012, pp. 1193-1200.

[6] D. Hind, C. Li, M. Sumner, and C. Gerada, "Realising robust low speed sensorless PMSM control using current derivatives obtained from standard current sensors," in Proceedings of the IEEE International Electric Machines and Drives Conference (IEMDC), May 2017, pp. 1-6.

[7] B. Weber, G. Lindemann, and A. Mertens, "Reduced observer for anisotropy-based position estimation of PM synchronous machines using current oversampling," in Proceedings of the 8th IEEE International Symposium on Sensorless Control for Electrical Drives (SLED), Sep. 2017, pp. 121-126.

[8] Z. Chen, H. Zhang, W. Tu, G. Luo, D. Manoharan, and R. Kennel, "Sensorless control for permanent magnet synchronous motor in rail transit application using segmented synchronous modulation," IEEE Early Access, vol. 7, pp. 76669-76679, 2019.

[9] M. X. Bui, M. Faz Rahman, D. Guan, and D. Xiao, "A new and fast method for on-line estimation of $\mathrm{d}$ and $\mathrm{q}$ axes inductances of interior permanent magnet synchronous machines using measurements of current derivatives and inverter DC-bus voltage," IEEE Trans. Ind. Electron., vol. 66, no. 10, pp. 7488-7497, Oct. 2019.

[10] G. Lindemann, N. Himker, and A. Mertens, "Enhanced observer with adaptive reference frame for self-sensing control of PMSM," in Proceedings of the 10th IEEE International Symposium on Sensorless Control for Electrical Drives (SLED), Sep. 2019, pp. 1-6.

[11] N. Himker, G. Lindemann, and A. Mertens, "Iterative tracker for anisotropy-based self-sensing control of PMSM," in Proceedings of the 10th IEEE International Symposium on Sensorless Control for Electrical Drives (SLED), Sep. 2019, pp. 1-6.

[12] P. Nussbaumer, M. A. Vogelsberger, and T. M. Wolbank, "Induction machine insulation health state monitoring based on online switching transient exploitation," IEEE Trans. Ind. Electron., vol. 62, no. 3, pp. 1835-1845, Mar. 2015.

[13] L. Peretti, P. Sandulescu, and G. Zanuso, "Method and device for detecting the presence of a permanent magnet of a rotor of a synchronous machine," US patent US10 291 165B2, May 14, 2019.

[14] R. Antonello, L. Ortombina, F. Tinazzi, and M. Zigliotto, "Online stator resistance tracking for reluctance and interior permanent magnet synchronous motors," IEEE Trans. Ind. Appl., vol. 54, no. 4, pp. 3405-3414, Jul. 2018. 